\title{
Linkage mapping of murine homolog of the yeast SPT6 gene to MMU11B1
}

\author{
P.-W. Chiang, ${ }^{1}$ P.A. Baldacci, ${ }^{4}$ C. Babinet, ${ }^{4}$ S.A. Camper, ${ }^{2}$ D. Watkins-Chow, ${ }^{2}$ D.D. Baker, ${ }^{2}$ C.-H. Tsai, ${ }^{5}$ \\ S. Ramamoorthy, ${ }^{1}$ E. King, ${ }^{1}$ A.C. Slack, ${ }^{3}$ E. Fogel, ${ }^{3}$ G. Morahan, ${ }^{6}$ A. Ashworth, ${ }^{7}$ C.C. Blackburn, ${ }^{8}$ D.M. Kurnit ${ }^{1,2,3}$ \\ ${ }^{1}$ Department of Pediatrics, University of Michigan Medical Center, 1150 West Medical Center Drive, 3520 MSRBI, Box 0650, Ann Arbor, Michigan \\ 48109-0650 USA \\ ${ }^{2}$ Department of Human Genetics, University of Michigan, Medical Center, Ann Arbor, Michigan 48109, USA. \\ ${ }^{3}$ Howard Hughes Medical Institute, Ann Arbor, Michigan, USA \\ ${ }^{4}$ Unit de Biologie du Developement, U.R.A.C.N.R.S. 1960, Institut Pasteur, Paris, France \\ ${ }^{5}$ Department of Medicine, University of Toronto, Toronto, Canada \\ ${ }^{6}$ Walter and Eliza Hall Institute of Medical Research, Melbourne 3050, Australia \\ ${ }^{7}$ Chester Beatty Laboratories, Fullham Road, London, UK \\ ${ }^{8}$ Nuffield Department of Medicine, John Radcliffe Hospital, Oxford University, Oxford, UK
}

Received: 30 January 1996 / Accepted: 5 March 1996

We have cloned the murine and human homologs of a gene, SPT6, that mediates chromatin structure and transcriptional activity in yeast (Chiang et al. 1996). The mammalian SPT6 homologs are virtually identical as they share $>98 \%$ identity and $>99 \%$ similarity at the protein level. The derived amino acid sequences of these two genes both predict a 1726 aa protein with several known features, including a highly acidic 5'-region, and MAPK consensus sites. We mapped the human SUPT6H gene to 17q11.2 and show here that the murine Supt6h gene maps to the corresponding region of $11 \mathrm{~B} 1$.

SPT6 was originally identified in the yeast, Saccharomyces cerevisiae. Sixteen mutations that modulate transcription in $S$. cerevisiae were selected as suppressors of Ty insertions at HIS4 and LYS2, and were termed the SPT (suppressors of $T y$ ) family of genes. Three members of this family in yeast are known: TFIID, histone H2A, and histone H2B. There are two genetically distinct groups of STP genes (TFIID group and histone group), with SPT4, SPT5, and SPT6 belonging to the histone group (Winston 1992). These genes suppress the loss of transcriptional activation of the SUC2 gene caused by snf2, snf5, and snf6 mutations. The SPT5 and SPT6 proteins have highly negatively charged $5^{\prime}$ regions, consistent with the hypothesis that they function in the nucleus, binding to positively charged nucleoproteins to form complexes that mediate chromatin structure. The importance of this subfamily is emphasized by the observation that most double mutant combinations among the SPT4, SPT5, and SPT6 genes are lethal in haploid strains (Winston 1992).

The emb-5 gene in C. elegans (Nishiwaki et al. 1993) is a homolog of the yeast SPT6 gene. emb-5 is required for gastrulation and for the correct timing of gut precursor cell division. The $h c 61$ mutation, a recessive, temperature-sensitive, maternal-effect mutation in the $e m b-5$ gene, results in the premature entry of E cells at the 26-cell stage into the mitotic (M) phase of the cell cycle. In addition to this early embryonic cell-cycle control, emb-5 acts during post-embryonic development: growth of emb-5 mutants (hc61) at a nonpermissive temperature from the L1 stage yields adults whose gonadal size is only half of that of the wild type and in whom no mature oocytes are found in the proximal arms of the gonads.

Correspondence to: $\mathrm{P} . \mathrm{-W}$. Chiang
An intragenic DNA polymorphic marker flanking a dinucleotide repeat in an intron of the murine Supt6h gene was isolated by sequencing a PCR product from genomic DNA. The PCR primers were derived from the Supt6h cDNA sequence, and the expected size of PCR product from the cDNA sequence is $270 \mathrm{bp}$. The sequence of the forward primer is $5^{\prime}$-GACATCAGCATAGATTTGAAGGG-3' and that of the reverse primer is $5^{\prime}$-GGTGCCACTCTCAACCAATT-3'. The PCR program was as follows: denaturing, $92^{\circ} \mathrm{C}(30 \mathrm{~s})$; annealing, $58^{\circ} \mathrm{C}(30 \mathrm{~s})$; extension, $72^{\circ} \mathrm{C}(30 \mathrm{~s})$ for 29 cycles, followed by $10 \mathrm{~min}$ at $72^{\circ} \mathrm{C}$. The amplified PCR product from genomic DNA was larger that expected from the cDNA sequence, indicating the insertion of an intron. Upon sequencing the PCR product, an intron with a dinucleotide repeat was found. Primers flanking the dinucleotide repeat were synthesized (forward: 5'-GGATCACAAGGTCAAGCCTGGG-3'; reverse: 5'-GTGAACTCGGGTTGTCAGGGCTG-3').

Supt6h was mapped to mouse Chromosome (Chr) 11 with an intersubspecific backcross of DF/B- $d f l d f$ with Mus musculus castaneus. The polymorphic region was amplified with the PCR primers flanking the dinucleotide repeat mentioned above. The amplification products, 175 and 193 bp for CASA/Rk and DF/B- $d f / d f$ respectively, were separated on $3 \%$ agarose gels and visualized by ethidium bromide staining. The backcross, $(\mathrm{DF} / \mathrm{B}-d f / d f \times \mathrm{CASA} /$ $\mathrm{Rk}) \times \mathrm{DF} / \mathrm{B}-d f / d f$, was previously typed for over 20 loci on Chr 11 (Roller et al. 1995). An interval mapping strategy was used to place Supt6h approximately on Chr 11. A subset of 97 progeny mice that were typed previously in the interval Asgrl to Thra were typed for Supt6h and D11Mit35. No recombinants were detected between Supt6h and Tnfaipl. Minimization of recombination unambiguously placed Supt6h with the following gene order and genetic distance: centromere-Asgrl, Rpo2-1-1.0 $\pm 1.0 \mathrm{cM}-$ (Tnfaip1, Supt6h)-2.1 $\pm 1.4 \mathrm{cM}-D 11$ Mit35-3.1 $\pm 1.8 \mathrm{cM}-T c f 2$. These results put Supt6h in a region of mouse Chr $11 \mathrm{~B} 1$ that exhibits extensive synteny homology with human Chr 17.

This subregional mapping on Chr 11 raised the possibility that variations in Supt6h could underlie the $\mathrm{Om}$ (ovum mutant) phenotype. This phenotype is marked by the lethality of embryos conceived by the mating of DDK females with males of other strains, whereas the mating of DDK males with females of other strains gave normal-sized litters (Baldacci et al. 1992). The involvement of the SPT6-homolog emb-5 in embryogenesis of $C$. elegans supported the hypothesis that mutations in Supt6h were 
responsible for the $\mathrm{Om}$ phenotype. This possibility was investigated formally by following segregation of Om and Supt6h in the backcross progeny of appropriate mating.

$\mathrm{BALB} / \mathrm{c}$ and DDK mice were from breeding colonies at the Pasteur Institute. The backcross between BALB/c females and $(\mathrm{BALB} / \mathrm{c} \times \mathrm{DDX}) \mathrm{F}_{1}$ males was described (Baldacci et al. 1992). This backcross was continued to the $\mathrm{BC} 6$ generation by mating males, heterozygous at the Om locus, to BALB/c females (Baldacci et al. 1995); $405 \mathrm{BC}$ individuals were analyzed. The genotype of recombinant backcross males at the $\mathrm{Om}$ locus was determined by an in vitro assay, as described previously (Baldacci et al. 1992). The EUCIB backcross has been described previously (Breen et al. 1994). The LS series $\left((\mathrm{SPR} \times \mathrm{C} 57 \mathrm{~B} 1 / 6) \mathrm{F}_{1} \times \mathrm{SPR}\right)$ contains 427 samples of which 207 are recombinants on Chr 11 between the markers Csfgm and D11Mit10. DNAs from 201 recombinant samples were analyzed with microsatellites in the region around $\mathrm{Om}$. The distances in the backcrosses were calculated as the frequency of recombinants with the $95 \%$ confidence interval of the distance given in square brackets.

Eight out of 240 progeny carried recombinations between Supt $6 h$ and $O m$, demonstrating that Supt6h was nonallelic, being $3.3 \mathrm{cM}[1.45-6.46 \mathrm{cM}]$ proximal to Om. Supt6h was not separated from the markers Nos2, D11Mit94, 96, and 144 in this cross. These markers are therefore at a maximum of $1.25 \mathrm{cM}$ from Supt $6 h$ at the $95 \%$ confidence level. These data were integrated into the map of Chr 11 (Baldacci et al. 1995). The order of markers and the genetic distances at the $95 \%$ confidence interval, calculated from the combined data are (oriented from $11 \mathrm{cen} \rightarrow$ tel):Supt6h, D11Mit94, 96, 144-1.67 cM [0.5-4.2 cM]-D11Mit118-0.85 cM [0.2-2.5 cM]D11Mit33, 93, Scya2-0.28 cM [0.0-1.6 cM]-Scyal, Om-1.55 [0.6-3.3 cM]-D11Mit35, 36-0.26 cM [0.0-1.4 cM]-D11Mit38, 39, Mpo.

The segregation of Spth6 and several Chr 11 markers was also tested on the EUCIB backcross to obtain an independent map and more precise mapping. The calculated distances between the markers were: Csfgm-8.4 cM [6.0-11.5 cM]-D11Mit30-0.23 cM [0.0$1.3 \mathrm{cM}]-A t p 1 b 2$, D11Mit31-1.4 cM [0.5-3.0 cM]-Supt6h, Nos2, D11Mit94, 96, 144-0.23 cM [0.0-1.3 cM]-D11Mit92, 95-0.47 cM [0.0-1.7 cM]-D11Mit33, 247-0.47 cM [0.0-1.7 cM]D11Mit37, Scyal, Scya2-0.47 cM [0.0-1.7 cM[-D11Mit36-36.5 cM [32-41.3 cM]-D11Mit10. The absence of recombinants in the 427 samples analyzed indicated that Supt $6 \mathrm{~h}$ is at a maximum of 0.7 cM (95\% confidence level) from the markers Nos2, D11Mit94, 96, and 144. In summary, fine mapping shows that Supt6h is not the Om locus, but it maps Supt6h more precisely on mouse Chr 11.

These genetic data indicated that Supt6h mapped into the region bounded by D11Mit31-D11Mit92 and that it was tightly linked to Nos2, D11Mit94, 96, and 144. Nos2, D11Mit94, 96, and 144 are all located on a YAC contig that spans most of the region D11Nds1 to D11Mit8 (C.C. Blackburn, G. Morahan, and A. Ashworth, unpublished; Nehls et al. 1994). YACs were identified by PCR screening of YAC DNA pools from the St. Mary's Hospital mouse YAC library and the Princeton University YAC library resource, or were obtained from Research Genetics (Huntsville, Ala.). The resulting YAC contig (C.C. Blackburn, G. Morahan, and A. Ashworth, unpublished) was probed by PCR with the Supt6h specific primers described above. PCR amplifications were performed with $\gamma-\left[{ }^{32} \mathrm{P}\right]$ ATP (Amersham) end-labeled primers as described (Blackburn et al. 1994). Reactions were run in a final volume of $10 \mu \mathrm{l}$ under the following PCR conditions: denaturing, $94^{\circ} \mathrm{C}(60 \mathrm{~s})$; annealing, $55^{\circ} \mathrm{C}(60 \mathrm{~s})$; extension, $72^{\circ} \mathrm{C}(30 \mathrm{~s}) ; 35$ cycles, before analysis on $4 \%$ denaturing TBE-polyacrylamide gels, followed by autoradiography. This analysis showed that Supt6h was present on YAC 61F12 but not on YAC 159D9, placing it between markers D11Bhm153 and D11Bhm154 (Nehls et al. 1995).
The mouse Supt6h gene maps into a region of considerable interest on MMU11, tightly linked to the mutations nude ( $\mathrm{nu}$; Takahasi et al. 1992), open eyelids (oe; Kelton and Rauch 1968), and ovum mutant (Om; Baldacci et al. 1992). The pleiotropic function described for homologs of this gene in lower eukaryotes suggests that Supt $6 h$ would be a candidate for any of these mutations. Our data clearly exclude Supt6h as a candidate for $\mathrm{Om}$, as Supt6h was segregated from this phenotype in an $O m$ backcross. Other investigators have screened this region of MMU11 for novel genes using both direct cDNA selection and exon trapping techniques. Using direct cDNA selection, Segre and associates (1995) detected a cDNA fragment with homology to $e m b-5$ on a mouse YAC that also contained the nude locus. Their data placed what we now know to be Supt6h approximately $100 \mathrm{~kb}$ from whn, which was recently identified as the candidate for the nude mutation (Nehls et al. 1994). This serendipitous finding is consistent with our extensive genetic and physical mapping, which mapped the Supt6h gene to YAC 61F12, within the minimum genetically defined nude locus (Segre et al. 1995). 61F12 also carries the whn gene (Nehls et al. 1994). The potential involvement of Supt6h in the open eyelids phenotype is being investigated.

\section{References}

Baldacci, P., Richoux, V., Renard, J.-P., Guénet, J.-L., Babinet, C. (1992). The locus $\mathrm{Om}$, responsible for the DDK syndrome, maps close to Sigje on mouse chromosome 11. Mamm. Genome 2, 100-105.

Baldacci, P.A., Cohen-Tannoudji, M., Kress, C., Pournin, S., Babinet, C. (1996). A high resolution map around the locus $\mathrm{Om}$ on mouse chromosome 11. Mamm. Genome, in press.

Blackburn, C.C., Griffith, J., Morahan, G. (1994). A high resolution map of the chromosomal region surrounding the nude gene. Genomics 26, 301317.

Breen, M. et al. (1994). Towards high resolution maps of the mouse and human genomes-a facility for ordering markers to $0.1 \mathrm{cM}$ resolution. Hum. Mol. Genet. 3, 621-627.

Chiang, P.-W. et al. (1996). Identification and analysis of the Lumen and murine putative chrometin structure regulator, SUPT6H and Supt6h. Genomics, in press.

Kelton, D.E., Rauch, H. (1968). Linkage of open eyelids with linkage group VII of the mouse. J. Hered. 59, 27-28.

Nehls, M., Pfeifer, D., Schorpp, M., Hedrich, H., Boehm, T. (1994a). New member of the winged-helix protein family disrupted in mouse and rat nude mutations. Nature 372, 103-107.

Nehls, M., Luno, K., Schorpp, M., Krause, S., Matysiak-Scholze, U., Prokop, C.-M., Hedrich, H.J., Boehm, T. (1994b). A yeast artificial chromosome contig on mouse chromosome 11 encompassing the $n u$ locus. Eur. J. Immunol. 24, 1721-1723.

Nehls, M., Luno, K., Schorpp, M., Pfeifer, D., Krause, S., MatysyakScholze, U., Dierbach, H., Boehm, T. (1995). YAC/ P1 contigs defining the location of 565 microsatellite markers and several genes across a 3.4-cM interval on mouse chromosome 11. Mamm. Genome 6, 321-331.

Nishiwaki, K., Sano, T., Miwa, J. (1993). emb-5 a gene required for the correct timing of gut precursor cell division during gastrulation in Caenorhabditis elegans, encodes a protein similar to the yeast nuclear protein SPT6. Mol. Gen. Genet. 239, 313-322.

Roller, M.L., Lossie, A.C., Koken, M.H.M., Smit, E.M.E., Hagemeijer, A., Camper, S.A. (1995). Localization of sequences related to the human RAD6 DNA repair gene on mouse Chr 11 and 13. Mamm. Genome 6, 305-306.

Segre, J.A., Nemhauser, J.L., Taylor, B.A., Nadeau, J.H., Lander, E.S. (1995). Positional cloning of the nude locus: physical and transcription maps of the region and mutations in the mouse and rat. Genomics 28, 549-559.

Winston, F. (1992). Analysis of SPT genes: a genetic approach toward analysis of TFIID, histones, and other transcription factors of yeast. In Transcriptional Regulation, (Cold Spring Harbor, N.Y.: Cold Spring Harbor Laboratory Press), pp. 1271-1293. 